\title{
Outcomes Bronchoscopic Evaluation in A University Hospital
}

\author{
Ram Hari Ghimire, ' Narendra Bhatta, Puru Koirala, 'Bides Bista, 'Deebya Raj Misra, 'Bhupendra Shah' \\ 'Divison of Pulmonary Critical Care and Sleep Medicine, Department of Medicine, BP Koirala Institute of Health Sciences, \\ Dharan, Nepal.
}

\section{ABSTRACT}

Introduction: Study of clinical profile of the patients and diagnostic yield of the selected bronchoscopic procedures gives us important information in clinical decision making and better patient care. There are hardly very few studies regarding these entities. Therefore, we decided to study clinical characteristics and outcomes of the patients who underwent bronchoscopic evaluation in our setting.

Methods: This was a cross-sectional study the consecutive patients who underwent bronchoscopy from $1^{\text {st }}$ May 2013- 30 $0^{\text {th }}$ April 2015 in division of pulmonary, critical care and sleep medicine. The main procedure performed was bronchoalveolar lavage.

Results: The mean age was 54.71 years with 76 (76\%) males. Recurrent hemoptysis in 58 (58\%) patients were the commonest indication. Total 95 (95\%) patients have chest X-ray abnormalities. The commonest bronchoscopic finding was bronchiectasis $23(23 \%)$ of patients followed by chronic bronchitis in 18 (18\%) and endobronchial tuberculosis in $16(16 \%)$. Total $10(71 \%)$ of the 14 bronchoscopically suspected lung cancer patients have intraluminal lesions. Bronchoalveolar lavage culture for tuberculosis showed growth in $46(46 \%)$, positive for malignancy in $7(7 \%)$ positive Ziehl Neelson stain for tuberculosis in $6(6 \%)$.

Conclusions: Bronchoscopic evaluation of patients with pulmonary diseases gives us a lot of information that may help us in better patient care and bronchoalveolar lavage has high diagnostic yield in diagnosing pulmonary tuberculosis.

Keywords: bronchoalveolar lavage;clinical profile;fiberoptic bronchoscopy.

\section{INTRODUCTION}

Pulmonary medicine has gone through a series of developmental stages over the last few decades since the invention of the bronchoscope by Gustav Killian ${ }^{1}$ in 1897 in Germany and the commercial introduction of the same by Ikeda $^{2}$ in 1967 . Now, bronchoscopy has gained widespread recognition in the field of respiratory medicine in diagnosing many pulmonary diseases. This is very safe and simple procedure for both diagnostic and therapeutic purposes.

The procedure nicely visualizes tracheobronchial tree and provides us spot diagnosis in many diseases. ${ }^{3}$ Study of clinical profile of the patients and diagnostic yield of the bronchoscopic procedures gives us important information in clinical decision making and better patient care.

There are very few studies regarding clinical profile and diagnostic utility of bronchoscopic procedure in these

Correspondence: Dr. Ram Hari Ghimire, Divison of Pulmonary Critical Care and Sleep Medicine, Department of Medicine, BP Koirala Institute of Health Sciences, Dharan, Nepal. Email: ramarogya 13@gmail. com, Phone: +977-9852027900. 
group of patients who underwent such evaluation in our setting. Hence, we aimed to study clinical profile and clinical utility of the bronchoscopy in these patients.

\section{METHODS}

This was a cross-sectional study of patients who underwent bronchoscopic evaluation consecutively in the division of pulmonary, critical care and sleep medicine of B.P Koirala institute of health sciences, Dharan, Nepal from $1^{\text {st }}$ May 2013- $30^{\text {th }}$ April 2015. Ethical Approval was taken. We reviewed the case file from medical record section (MRD), Bronchoscopic suite and retrieved the reports of bronchoalveolar lavage (BAL) from Clinical Lab Services and Tuberculosis Laboratory. A structured proforma was prepared to include patient demographics, indications, X-ray findings, prebronchoscopic radiological as well as clinical diagnosis, bronchoscopic findings, procedures performed, sample types, types of investigation sent and their reports and any complications during procedure. The data was not included in analysis with incomplete procedure and incomplete records from any reason. Ethical clearance was obtained before doing the study.

The collected data was entered in Microsoft Excel 2013 and converted into Statistical Software Package for Social Sciences (SPSS 11.5 version) for statistical analysis for descriptive statistics. Descriptive statistics was used for analysis.

\section{RESULTS}

After the analysis of the clinical characteristics and outcomes of the patients who underwent bronchoscopic evaluation, we found the following results.

The minimum age was 18 and maximum was 85 with mean age of $54.71 .65(65 \%)$ of the patients were above 50. Majority 76 (76\%) patients were males (Figure 1).

Indications of Bronchoscopy: Commonest indication for bronchoscopic study was recurrent hemoptysis in 58 $(58 \%)$ patients followed by chronic cough in 30 (30\%) and non-resolving pneumonia in $12(12 \%)$.

Pre-bronchoscopic Chest X-ray diagnosis: In 95 $(95 \%)$ of the patients, the chest X-ray was abnormal. Bronchiectasis and PTB were most common in bilateral upper zones. Lung mass was more common in right middle zone followed by right upper zone, left lower zone. Pneumonia was more common in right lower zone (Table 2). Other associated findings in prebronchoscopic chest X-ray were collapse in 12 (12\%), cavity in $6(6 \%)$, lung abscess in $(3 \%)$ and pleural effusion in $(3 \%)$.

\begin{tabular}{|lc|}
\hline Table 1. Chest X-ray diagnosis. \\
\hline Lung lesion & $\mathbf{n}(\%)$ \\
Lung mass & $20(20)$ \\
Pulmonary Tuberculosis & $20(20)$ \\
Pneumonia & $18(18)$ \\
Bronchiectasis & $37(37)$ \\
\hline
\end{tabular}

Among 14 suspected lung cancer patients, 10 (71\%) have intraluminal lesion and 4 (29\%) extraluminal lesion. Two patients have associated left vocal cord palsy and one had right vocal cord palsy.

\begin{tabular}{|lc|}
\hline Table 2. Bronchoscopic diagnosis. \\
\hline Lung Lesions & $\mathbf{n}(\%)$ \\
Bronchiectasis & $23(23)$ \\
Chronic bronchitis & $18(18)$ \\
Endobronchial Tuberculosis & $16(16)$ \\
Acute pneumonitis & $12(12)$ \\
Acute bronchitis & $8(8)$ \\
Lung mass & $14(14)$ \\
\hline
\end{tabular}

Results of bronchoalveolar lavage examination: Potassium hydroxide $(\mathrm{KOH})$ preparation for fungal elements were sent in $25(25 \%)$ patients, all were negative for fungal elements. BAL was sent for culture and sensitivity in $28(28 \%)$ patients with suspected persistent pulmonary infection other than tuberculosis. Pseudomonas auruginosa was grown in 7 (25\%) patients, cytrobacter species in one patient. All of them were sensitive only to imipenem and amikacin. All the samples were sent for culture for tubercular bacilli and Ziehl Neelson stain. Tubercular bacilli was grown in 46 (46\%) of the patients, Acid Fast Bacilli was positive in $6(6 \%)$. All the BAL smear positive patients were positive for culture for mycobacterium tuberculosis. Mycobacterium tuberculosis was grown in $44(44 \%)$ patients and remaining 2 (2\%) were mycobacterium bovis. The patients with lung cancer from bronchoscopic study were not positive for smear and culture for mycobacterium tuberculosis. Among 14 bronchoscopically suspected lung cancer patients, 7 (50\%) patients were positive malignancy, most have squamous cell carcinoma 6 (85\%), and 1 (14.2\%) had adenocarcinoma.

Complications: Mild respiratory distress was common in many elderly patients that resolved spontaneously on completion of the study and only one patient had procedure related nasal bleeding that subsidedon observation. There were no other major complications. 


\section{DISCUSSION}

Among 100 patients, we found that the mean age of the patients was 54.71 with range of $18-85$ years. Our result is close to Anandan PT et $\mathrm{al}^{5}$ who found mean age of 56.15 with range of $13-90$ years. Another study by Gaude GS et $\mathrm{al}^{6}$ found mean age of 47 years with range of $17-83 y e a r s . T h e$ mean age in this study ${ }^{6}$ was lower than ours probably because of large sample size (n-1116) in their study. Majority of our patients were males $(76 \%)$ that are consistent with the study by Gaude GS et al $^{6}$ where $71 \%$ were males but Devkota $\mathrm{KC}$ et $\mathrm{al}^{4}$ found that $65 \%$ were males and Anandan PT et $\mathrm{al}^{5}$ found $86 \%$ males in their study. In our study most of the patients were above fifty years that is similar to the another study. ${ }^{4}$

In our patients hemoptysis (58\%) was the commonest indication followed by chronic cough $(30 \%)$ and nonresolving pneumonia in $(12 \%)$ while Devkota et $\mathrm{al}^{4}$ found hemoptysis in $44.2 \%$, cough in $89.8 \%$. Sinha $\mathrm{S}$ et $\mathrm{al}^{7}$ found hemoptysis in $32 \%$. Barnes TW et $\mathrm{al}^{8}$ found chronic cough as the commonest symptom of patients undergoing bronchoscopy. Prakash UBS et $\mathrm{al}^{9}$ reported pulmonary infiltrate in $25 \%$, hemoptysis in $14 \%$, mass lesion in $12.2 \%$, hilar adenopathy in $10.9 \%$, pulmonary nodule in $10.7 \%$ patients. Anandan PT et al ${ }^{5}$ found pneumonia in $15.5 \%$ patients. Jainm $P$ et $\mathrm{al}^{10}$ showed non resolving pneumonia as indication in $18 \%$, hemoptysis in $15 \%$ lung mass in $48 \%$. Gaude GS et $\mathrm{al}^{6}$ reported the following indications: suspected PTB $(28.3 \%)$, suspected lung cancer in $26.1 \%$, suspected infection in $17.5 \%$, interstitial lung disease in $10.5 \%$, hemoptysis $4.6 \%$, connective tissue disease in $4.4 \%$, chronic cough in $2.9 \%$, hoarseness of voice in $2.4 \%$, atelectasis in ICU in $2.3 \%$, chest trauma $1 \%$. This percentage variation in indications in different studies may be due to lack of uniformity between researchers in methodology, choosing indications and difference in the centers where studies were performed.

In our study $95 \%$ of the x-rays were abnormal before procedure and this is close to the other studies. ${ }^{4,5}$ In our study bronchiectasis was the commonest diagnosis $(37 \%)$ followed by lung mass $(20 \%)$, pulmonary tuberculosis (20\%) and pneumonia (18\%). Bronchiectasis and PTB are most common in bilateral upper zones than other zones in chest X-ray. Lung mass was more common in right middle zone. Pneumonia was more common in right lower zone. Anandan PT et al s study ${ }^{5}$ has $x$-ray finding of lung mass in $49 \%$ that was common in right upper zone followed by left upper zone and left mid zone, ILD in $1.6 \%$, and pneumonia in $15.5 \%$, collapse in $8.7 \%$, bronchiectasis in $2.3 \%$, others in $22 \%$. Devkota $\mathrm{KC}$ et $\mathrm{al}^{4}$ found radiological opacity in $90.2 \%$, hemoptysis with normal chest X-ray in $1.7 \%$, diffuse pulmonary infiltrate in $4.3 \%$ and others in $3 \%$. Khara NV et al ${ }^{11}$ reported cavitory lesion in $5.5 \%$, collapse in $10 \%$, consolidation in $27 \%$, infiltration and fibrosis in $27 \%$, mass lesion in $23.5 \%$, nodular lesion in $2.4 \%$, reticular pattern in $0.3 \%$ and normal $X$-rayin $3.5 \%$ in their pre-bronchoscopic chest $x$-ray findings. In our study bronchiectasis was mostly found in upper lung zones that may be because of high prevalence of pulmonary tuberculosis and its sequelae in our country. The similarity and dissimilarity in pre-bronchoscopic finding in chest $x$-ray between different studies may be due to lack of uniformity in reporting the $x$-ray films, inter-observer variation in reading them and lack of uniformity in choosing the indication for bronchoscopy.

In our study bronchiectasis was the most common diagnosis in $23 \%$ patients followed by lung cancer $(14 \%)$, chronic bronchitis $(18 \%)$, endobronchial tuberculosis $(16 \%)$, acute pneumonitis $(12 \%)$ and acute bronchitis (8\%), 10 lung cancer patients (71\%) have intraluminal luminal lesion, extraluminallesion in 4 $(28 \%)$, two patients have associated left vocal cord palsy and one had right vocal cord palsy. Devkota et $\mathrm{al}^{4}$ found that $47.8 \%$ patients have intraluminal lesions, suspected growth in $4 \%$ and non-specific inflammation in 10.2\%, inconclusive in $26.1 \%$ and normal in $11.9 \%$. We found normal bronchoscopy in $9 \%$. There is difference in findings between two studies it is probably because of under reporting of the specific inflammatory conditions by them. The study by Anandan PT et $\mathrm{al}^{5}$ found left vocal cord palsy in $7.7 \%$, right vocal cord palsy in $2.6 \%$, intraluminal growth in $26.8 \%$, extraluminal compression in $28.6 \%$, inflammatory lesion in $29.6 \%$ and normal in $18 \%$ and others in $1.9 \%$. Our results tend to correlate with Anandan PT et al's probably because of similarity in patient population and geography.

Any patient with pulmonary infiltrates, BAL can be very good mode for investigating lower respiratory tract. Recovering BAL is a very simple and straight forward technique with least complication in experienced hands. Mycobacterium tuberculosis was grown in $46 \%$ of the patients, smear for Acid Fast Bacilli was positive in (6\%), lung cancer in (7\%), most are squamous cell carcinomas $(85 \%)$, and (14.2\%) had adenocarcinoma. Gaude GS et $\mathrm{al}^{6}$ diagnosed pulmonary tuberculosis by bronchoscopic evaluation in $42.5 \%$ patients. JacomelliM et $\mathrm{al}^{12}$ found that BAL has sensitivity of $60 \%$ and specificity of $100 \%$ in diagnosing tuberculosis in tuberculosis endemic countries. They diagnosed pulmonary tuberculosis in $44 \%$ patients by combining BAL and transbronchial biopsy (TBB). CharoentratanakulS et al ${ }^{13}$ performed BAL and TBB in 40 PTB suspected patients and they found positive BAL smear in $7.5 \%$ patients that is close to our study. The BAL culture was positive for mycobacterium 
tuberculosis in $15 \%$. Another study done by Dasgupta KS et al included 104 patients where mycobacterium tuberculosis positive in aspiration and washing smear in $38 \%$, positive mycobacterium culture alone in $27 \%$. Khara NV et al $^{11}$ studied BAL of 289 patients and found smear positive for tuberculosis in $4.5 \%$, fungal stainin $2.1 \%$ and cytology in $19.4 \%$ patients. Stephen I Rennard ${ }^{15}$ found that over all diagnostic yield of BAL for lung cancer was $50 \%$ range being $35-59 \%$, common cancers being squamous cell carcinoma and adenocarcinoma. Semenzato $\mathrm{G}^{16}$ found that overall diagnostic yield is $76 \%$ in patients with biopsy and autopsy proven neoplasms and $92 \%$ in bronchoalveolar carcinoma. Among 14 bronchoscopically suspected cases we found lung cancer in 7 patients (Diagnostic yield-50\%) and six of them were squamous cell carcinoma and one adenocarcinoma. Our findings are consistent with these authors findings.

One patient had nasal bleeding and some others had minor procedure related respiratory distress, these spontaneously resolved on observation. Devkota KC et $\mathrm{al}^{4}$ also noticed minor procedure related bleeding in few cases. Anandan PT et $\mathrm{al}^{5}$ reported hemorrhage in $5.7 \%$, respiratory distress in $0.7 \%$. Gaude GS et $\mathrm{al}^{6}$ noticed complications in $1.9 \%$.

\section{CONCLUSIONS}

Bronchoscopic evaluation of patients with pulmonary diseases gives us a lot of information that may help us in better patient care and bronchoalveolar lavage has high diagnostic yield in diagnosing pulmonary tuberculosis.

\section{ACKNOWLEDGEMENTS}

We thank Dharnidhar Baral, Biostatistician, Department of Community Medicine and Mr. Rupesh Kumar Shreewastav, Department of Biochemistryat BPKIHS.

\section{Conflict of Interest: None.}

\section{REFERENCES}

1. Killian G.Direct endoscopy of the upper air passages and esophagus: its diagnostic and therapeutic value in the search for removal of the foreign bodies. J Laryng. 1902;18:461.

2. Ikeda M. Flexible Fibroptic Bronchoscope. J JpnBronchoesophageal Soc. 1968;19-54.

3. 3.Kdvenat DM, Rath GS, Anderson WM,Snider GL.Maximal extent of visualization of bronchial tree by flexible fibreoptic bronchoscopy.Am Rev Respir Dis.1984;110:88-90.

4. Devkota KC, Pathak R, Khanal A, Chokhani R. Fiber-optic Bronchoscopy:Seven years experience at hospital Nepal Medical College teaching hospital. Nepal Med Coll J. 2010 ;12(4):260-3.

5. Anandan PT, Rajgopal TP, James PT, Ravindran C. Clinical profile of the patients undergoing Fiber Optic Bronchoscopy in a tertiary care setting. Indian J Bronchol. 2006 May-Aug 1:58.

6. Gaude GS, Mathew I. Diagnostic yield of flexible bronchoscopy in the current clinical practice in a tertiary care hospital. Indian J Bronchol. 2007 Jan-Apr;2:130:

7. Sinha S,Guleria R, Pande JN, Pandey RM. Bronchoscopy in adults at a tertiary care centre: indications and complications. J Indian Med Assoc. 2004 Mar;102(3):152-6.

8. Barnes TW, AfessaB.The clinical utility of Flexible Bronchoscopy in the evaluation of chronic cough. Chest. 2004;126:268-2.
9. Prakash UBS, Offord KP, Stubbs SE.Bronchoscopy in North America: the ACCP survey. Chest. 1991;100:1668-75.

10. Jainm P, SandurS.Role of Flexible Bronchoscopy in immunocompromised patients with lung infiltrates. Chest. 2004;125:712-22.

11. Khara NV, Kshatriya RM, Vala DH, Prajapati DN, Paliwal RP. Diagnostic yield of Fiberoptic Bronchoscopy in three common lung conditions at a rural teaching hospital. National JMed Research. 2013 Oct-Dec;3(4):392-5.

12. Jacomelli M, Silva PR, Rodrigues AJ, Demarzo SE, Siecento M, Figueiredo VR, et al. Bronchoscopy for the diagnosis pulmonary tuberculosis in patients with negative sputum smear microscopy results. J Bras Pneumol. 2012; March-Apr;38(2):167-73.

13. Charoentratanakul S, Desjomritrutai W, Chaiprasert A Diagnostic role of fiberoptic bronchoscopy in suspected smear negative pulmonary tuberculosis. Respir Med. 1995;89:621-3.

14. Dasgupta KS, Mundada PS, Soni N. Diagnostic role of fiberoptic bronchoscopy in pulmonary tuberculosis. Indian J Otolaryng and Head and NeckSurg. 2000 Oct-Dec;52(4):347-9.

15. Stephen IR. Bronchoalveolar lavage in diagnosis of cancer. Lung. 1990. Supplement 1035-40.

16. Semenzato G, Polleti V.Bronchoalveolar lavage in lung cancer. Respiration 1992; Supplement 159:44-6. 